\title{
Emotional Semantic Calculation Model and Retrieval of Wedding Image based on Color Features
}

\author{
Qiaoyan LIU ${ }^{1}$, Yi SUN² \\ ${ }^{1}$ School of Software Engineering, Beijing University of Posts and Telecommunications, Beijing, \\ 102209, China \\ ${ }^{2}$ School of Software Engineering, Beijing University of Posts and Telecommunications, Beijing, \\ 102209, China \\ 1email: 490949213@qq.com, ²email:sunyi@bupt.edu.cn
}

\begin{abstract}
Keywords: Wedding Image; Emotional Semantic; Image Classification; Image Retrieval; Color Feature; Fuzzy Function; Crossing Method
\end{abstract}

\begin{abstract}
Content-based retrieval is now commonly used in image retrieval system. One of the disadvantages of the traditional content-based image retrieval technology is the "semantic gap", which means could not get the semantic meaning from the basic image features. In the context of wedding image, considering the subjectivity of emotion, this paper will present a further research on emotion semantic extracting of wedding image based on color features. Firstly, we will extract the color eigenvector of images using asymmetrical quantized color histogram, and then propose an optimized formula, which helps to classify colors for better visual characteristic. Secondly, five basic emotional classes will be put forward, along with a mapping model that calculates images' emotional eigenvector by using fuzzy function based on the five classes. Moreover, the use of crossing method to measure similarity degree between images will also be applied. Finally, we will carry out retrieval experiments whose recall ratio and precision ratio indicate the correctness and effectiveness of this system.
\end{abstract}

\section{Introduction}

For a wedding, colors establish emotional tone. We want to extract the emotion semantic of a wedding image, instead of texturing and shaping features which are less meaningful in wedding emotion expression, thus we can retrieve similar images based on emotion semantic in order to find appropriate wedding planning companies. Although the background of a wedding is complicated, it is easy to extract its color features because of its impregnability by the size and direction of image. At present, the most commonly used color space is HSV(hue, saturation, value), and the most commonly used color extracting method is global color histogram.

In this paper, image emotional semantic extracting will be discussed from three aspects. First, extract the color eigenvector of images using color histogram. Considering the similarity of adjacent color unit which composing color eigenvector, we propose an optimized formula, which helps to classify colors better. Second, we put forward five basic emotional classes, and implement a mapping model which switch color feature to emotion semantic using fuzzy function. This model can calculate the emotion semantic scores of each color unit, then figure out emotional eigenvector of whole image based on the five classes. Inspired by the crossing method used in color histogram, this method is applied to calculate emotional similar degree between images. Finally, we evaluate the performance of this retrieval system with recall ratio and precision ratio. Experiments demonstrate the correctness and effectiveness of this system.

\section{Image Color Eigenvector Extracting}

\subsection{Asymmetrical Quantized Color Histogram}

We first extract images' color eigenvector using color histogram. HSV color space is chosen to describe colors because it suits people's visual characteristic better. This method is used widely so we only mention here briefly. We can get RGB of a pixel from an image, then we use certain formula to transform $(\mathrm{r}, \mathrm{g}, \mathrm{b})$ to $(\mathrm{h}, \mathrm{s}, \mathrm{v})$, next quantize (h,s,v) asymmetrically to decrease vector 
dimension. The asymmetrical quantization formula is as followed,

$$
\begin{aligned}
& H= \begin{cases}1 & \text { if } h \in[23,50] \text { orange } \\
2 & \text { if } h \in[51,75] \text { yellow } \\
3 & \text { if } h \in[76,155] \text { green } \\
4 & \text { if } h \in[156,180] \text { cyan } \\
5 & \text { if } h \in[181,275] \text { blue } \\
6 & \text { if } h \in[276,290] \text { purple } \\
7 & \text { if } h \in[291,315] \text { purlish red } \\
0 & \text { else red }\end{cases} \\
& S=\left\{\begin{array}{ll}
0 & \text { if } s \in[0,0.2] \\
1 & \text { if } s \in(0.2,0.7] \\
2 & \text { if } s \in(0.7,1]
\end{array} \quad V= \begin{cases}0 & \text { if } v \in[0,0.2] \\
1 & \text { if } v \in(0.2,0.7] \\
2 & \text { if } v \in(0.7,1]\end{cases} \right.
\end{aligned}
$$

Next we use formula(4) to composite three-dimensional vector(h,s,v) into one-dimensional vector L. As follows, $Q_{S}$ and $Q_{V}$ is the quantitative series of $\mathrm{S}$ and $\mathrm{V}$. We know $Q_{V}=3, Q_{S}=3$ from formulas above, thus $\mathrm{H}, \mathrm{S}, \mathrm{V}$ is spread out in one-dimensional vector.

$$
L=Q_{S} Q_{V} H+Q_{V} S+V=9 H+3 S+V \quad L \in[0,71]
$$

Finally calculate the frequency of each color unit in color histogram $\mathrm{L}$, then we get the color eigenvector $Q\left(q_{0}, q_{1}, q_{2} \ldots q_{L}\right)$.

\subsection{Optimized Color Classification}

In traditional color histogram, quantization boundary decides the classification of color, when dealing with a pixel, it is common to classify it into one color unit of color eigenvector, but this color also has similarity with adjacent color units in a way. The research in part 3 requires us to transform a color unit into one specific visible color, considering the similarity between color units, we propose an optimized formula to classify colors better.

Two figures are provided to show the change law in color units. In Figure1-2, When $S=0$, hue is meaningless and always equal to 0 , at this time the color is most shallow and is decided by luminance. There are 72 eigenvalues of color units in a color eigenvector. Black, gray, white pixels are all counted in hue $=0$ (red) section, so we have 51 actual colors in total.

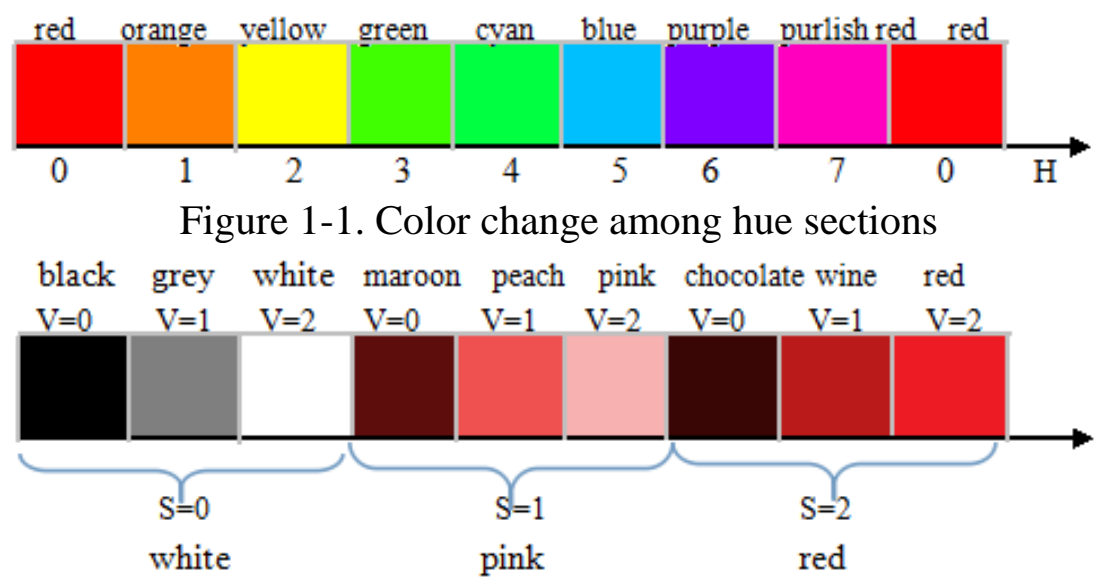

Figure 1-2. Color change in red hue section

Research shows that the influence caused by luminance and saturation exceed by hue when consider the expression of color emotion. So we only need to consider the similarity between colors that have different hue, same luminance and saturation. Through a lot of experiments we get the best contribution rate as followed.

For a pixel whose hue $=\mathrm{i}$, luminance $=\mathrm{v}$, and saturation $=\mathrm{s} \quad(s \neq 0), \mathrm{d}$ is the shortest distance from $\mathrm{j}$ to $\mathrm{i}$ in hue loop which is shown in Figure 1-1. The contribution rate to color units in all hue sections 
$Q_{S} Q_{V} j+Q_{V} s+v \quad \mathrm{j} \in[0,7]$ is

$$
\left(\frac{1}{2}\right)^{d^{2}+1}
$$

In this way, the contribution rate is smaller when $d$ is bigger. Experiments show that color classification carried out with this formula is more suitable to subjective feeling.

\section{Emotional Semantic Calculation Model based on Fuzzy Membership Function}

\subsection{Fuzzy membership function based on Statistical Method}

Fuzzy Theory can represent people's experience and common sense in an appropriate computer form, it was proved to be an accurate method when extracting image emotional semantic [2].

5 emotional classes are put forward as fundamental emotion units in wedding emotion expressing. A is fiery-festive, $\mathrm{B}$ is warm-pleasant, $\mathrm{C}$ is romantic-dreamlike, $\mathrm{D}$ is fresh-natural and $\mathrm{E}$ is classical-dignified. Number 72 color units in [0,71] sequentially, and let all color units be the domain of discourse except for black, gray and white. Then use statistical method to gain membership function which suit the subjective feeling of most people better.

In order to calculate the membership degree of $\mathrm{X}$ to $\mathrm{A}$..E, we carried out $\mathrm{N}$ times independent repeated experiments. In each experiment, user select several color units from 48 colors which they think belong to set A...E, and the same color unit can be selected more than once. Let $A_{*}$ be the color unit set which is decided to belong to $\mathrm{A}$ in one experiment, as for color unit $x_{k}, \mathrm{M}$ is the times when $x_{k} \in A_{*}$, if $\mathrm{N}$ is large enough

$$
\mu_{A}\left(x_{k}\right)=\frac{M}{N}
$$

$\mathrm{X}$ is the Domain of Discourse, $\mu_{A}(x)$ is the membership degree of $\mathrm{x}$ to set A.

We issue questionnaire in several well-known websites and finally get 402 valid results. Some results are shown in Table 2-1, and the analysis result is in Table 2-2.

Table 2-1 Computed membership degree of some hue sections (Unit: \%)

\begin{tabular}{|c|c|c|c|c|c|c|}
\hline \multicolumn{7}{|c|}{ Hue $=2$ (Yellow) } \\
\hline & 21 & 22 & 23 & 24 & 25 & 26 \\
\hline A & 7.2 & 5.4 & 10.7 & 0 & 0.5 & 17.3 \\
\hline B & 0 & 20.5 & 55.0 & 0 & 12.3 & 80.1 \\
\hline C & 6.1 & 0 & 8.0 & 0 & 0 & 10.5 \\
\hline D & 0 & 0 & 3.1 & 0 & 0.9 & 5.3 \\
\hline E & 53.3 & 3.8 & 0 & 40.1 & 2.4 & 0 \\
\hline \multicolumn{7}{|c|}{ Hue $=3$ (Green) } \\
\hline & 30 & 31 & 32 & 33 & 34 & 35 \\
\hline A & 0 & 0 & 0 & 0 & 0 & 0 \\
\hline B & 0 & 1.4 & 0 & 0 & 0 & 3.2 \\
\hline C & 0 & 0 & 2. 0 & 0 & 0.2 & 7.0 \\
\hline D & 0 & 40.9 & 55.7 & 0 & 80.0 & 81.1 \\
\hline E & 13.3 & 4.0 & 0 & 20.6 & 0 & 0 \\
\hline
\end{tabular}

\begin{tabular}{|c|c|c|c|c|c|c|}
\hline \multicolumn{7}{|c|}{ Hue $=\mathbf{0}$ (Red) } \\
\hline & $\mathbf{3}$ & $\mathbf{4}$ & $\mathbf{5}$ & $\mathbf{6}$ & $\mathbf{7}$ & $\mathbf{8}$ \\
\hline $\mathbf{A}$ & 0.2 & 51.4 & 10.7 & 0 & 50.5 & 67.3 \\
\hline B & 0 & 40.4 & 30.0 & 5.2 & 12.3 & 50.1 \\
\hline C & 1.0 & 3.7 & 57.9 & 0 & 4.2 & 5.0 \\
\hline D & 0 & 0 & 8.0 & 0 & 0 & 0 \\
\hline E & 53.3 & 3.3 & 0 & 34.6 & 0.4 & 0 \\
\hline \multicolumn{7}{|c|}{ Hue=1 (0range) } \\
\hline & $\mathbf{1 2}$ & $\mathbf{1 3}$ & $\mathbf{1 4}$ & $\mathbf{1 5}$ & $\mathbf{1 6}$ & $\mathbf{1 7}$ \\
\hline A & 0 & 0 & 30.5 & 0 & 5.5 & 46.3 \\
\hline B & 0 & 14.4 & 61.0 & 0.8 & 61.6 & 78.2 \\
\hline C & 0 & 6.7 & 27.1 & 0 & 5.2 & 7.0 \\
\hline D & 0 & 3.2 & 9.7 & 0 & 0 & 8.1 \\
\hline E & 33.3 & 4.3 & 0 & 40.6 & 31.4 & 0 \\
\hline
\end{tabular}


Table 2-2 Distribution of color units which have high membership degree

\begin{tabular}{|c|c|}
\hline & $\begin{array}{c}\text { Hue sections where color units have } \\
\text { high membership degree }(>50 \%) \text { to class } \mathrm{X}\end{array}$ \\
\hline $\begin{array}{c}\text { A } \\
\text { fiery-festive }\end{array}$ & $\begin{array}{c}\text { 0(red), } 7 \text { (purlish red) } \\
\text { where saturation and luminance are both high }\end{array}$ \\
\hline $\begin{array}{c}\text { B } \\
\text { warm-pleasant }\end{array}$ & $\begin{array}{c}\text { 2(orange), 3(yellow) } \\
\text { where saturation and luminance are both high }\end{array}$ \\
\hline $\begin{array}{c}\text { C } \\
\text { romantic-dreamlike }\end{array}$ & $\begin{array}{c}\text { 0(red), 5(blue), 6(purple) } \\
\text { where saturation and luminance are both high }\end{array}$ \\
\hline $\begin{array}{c}\text { D } \\
\text { fresh-natural }\end{array}$ & $\begin{array}{c}\text { 3(green), 4(cyan), 5(blue) }) \\
\text { where saturation and luminance are both high }\end{array}$ \\
\hline $\begin{array}{c}\text { E } \\
\text { classical-dignified }\end{array}$ & low luminance(V) of some hue sections \\
\hline
\end{tabular}

3.2 Emotional Semantic Mapping Model

$$
S_{A}=\sum_{i=t}^{N} C_{i} \times \mu_{A}\left(x_{i}\right)
$$

$S_{A}$ represents the emotional score of set $\mathrm{A}, \mathrm{N}$ is the number of color units, $C_{i}$ is the proportion of color unit $\mathrm{i}$ in image color histogram, $\mu_{A}\left(x_{i}\right)$ is the membership degree of color unit $\mathrm{i}$ to A. Apply this formula to all fuzzy sets, after normalization we get the emotional eigenvector $E\left(e_{A}, e_{B}, e_{C}, e_{D}, e_{E}\right)$ of a image, thus implement the mapping from color to emotion.

\subsection{Image Similarity Measurement Method based on Emotional Eigenvector}

Inspired by the crossing method used in color histogram, we can use it to calculate emotional similar degree between images too. Let $\mathrm{Q}$ be an example image which emotional eigenvector is $E\left(e_{A}, e_{B}, \ldots, e_{E}\right)$ while image $\mathrm{H}$ in database is $H\left(h_{A}, h_{B}, \ldots, h_{E}\right)$, and e, h are all normalized to $[0,1]$, the emotional similar degree can be expressed as formula(8) which describe the common part of two images.

$$
\operatorname{Sim}(E, H)=\sum_{i=A}^{F} \min \left(e_{i}, h_{i}\right)
$$

\section{Performance Analysis}

Recall ratio and precision ratio are evaluation standards in information retrieval which are now commonly used in image retrieval. The definitions are

$$
\begin{aligned}
& \text { Recall ratio }=\frac{\text { number of images which are retrieved correctly }}{\text { number of images which should be retrieved }} \\
& \text { Precision ratio }=\frac{\text { number of images which are retrieved correctly }}{\text { number of images which are retrieved }}
\end{aligned}
$$

In order to evaluate the performance of retrieval system, we collect 100 wedding images and make a subjective investigation about these images so as to calculate the standard emotional eigenvector of each image. In each experiment, a user should select one or more emotional class $\{A, B, C, D, E\}$ for each image. Use formula(11) to calculate eigenvalue of each class $X$.

$$
s_{X}=\frac{N_{X}}{T N}
$$

$N_{X}$ is the number of people who choose class $\mathrm{X}$ for this image, TN is the total number of people. After normalization, we can get each image's standard eigenvector $S\left(s_{A}, s_{B}, \ldots, s_{E}\right)$.

The primary emotional class of an image is which has highest eigenvalue. We use 0.65 as the threshold value of similarity degree which is calculated using formula(8) when decide whether an image should be retrieved or not. The threshold value is flexible and can be adjusted according to 
users' willingness. Comparing retrieved results using our model with using the standard emotional eigenvector, the computed result is

\begin{tabular}{cccccc}
\hline $\begin{array}{c}\text { Primary } \\
\text { Class }\end{array}$ & $\begin{array}{c}\mathrm{A} \\
\text { fiery- } \\
\text { festive }\end{array}$ & $\begin{array}{c}\text { B } \\
\text { warm- } \\
\text { pleasant }\end{array}$ & $\begin{array}{c}\mathrm{C} \\
\text { romantic- } \\
\text { dreamlike }\end{array}$ & $\begin{array}{c}\mathrm{D} \\
\text { fresh- } \\
\text { natural }\end{array}$ & $\begin{array}{c}\mathrm{E} \\
\text { classical- } \\
\text { dignified }\end{array}$ \\
\hline Recall & 0.784 & 0.820 & 0.720 & 0.892 & 0.804 \\
\hline Precision & 0.940 & 0.866 & 0.928 & 0.815 & 1.000 \\
\hline
\end{tabular}

\section{Conclusion}

In conclusion, this system analyzed the emotional semantic that wedding image expressed, it's a beneficial exploration in the field of the research about emotional semantic application. The research is based on the perception about colors of most people and obtains a good result, but we should realize that individuality still exists, the system will be more thorough if we adjust individual membership degree of colors utilizing his/her feedback and improve the existing classification.

\section{References}

[1] Lihua Cao, Wei Liu, Guohui Li, The research and implementation of image retrieval algorithm based on multiple dominant hues, JOURNAL OF COMPUT ER RESEARCH\& DEVELOPMENT, Vol. 36, No. 1, Jan. 1999

[2] Weining Wang, Linying Yu, A Survey of Image Emotional Semantic Research, JOURNAL OF CIRCUITS AND SYSTEMS, Vol.8 No.5,October 2003

[3] Weining Wang, Image feature extracting, retrieval and classification based on emotional semantic, SOUTH CHINA UNIVERSITY OF TECHNOLOGY, April 1st , 2005

[4] Li Pingting, The Emotion Classification of House-Designing Image Based on Color Feature. June 4th , 2011

[5]HUANG Kun , LAI Mao-sheng, Classification and extraction of image affective features, Computer Applications, 1001-9081(2008)03-0659-03 